\section{Closure of a mucosal entry using the clip-with-line method}

\section{Hironari Shiwakua, Kanefumi Yamashitaa, Haruhiro Inoue ${ }^{b}$, Suguru Hasegawa ${ }^{a}$}

Fukuoka University, Fukuoka; Showa University Koto Toyosu Hospital, Tokyo, Japan

Peroral endoscopic myotomy (POEM) is a revolutionary therapy for achalasia $[1,2]$. Because incomplete closure of a mucosal entry may lead to severe mediastinitis after POEM, secure closure of this site is very important in clinical settings. However, unlike general surgery, endoscopic procedures are performed with one hand, and there is no countertraction of the mucosa. Therefore, slippage of clips or an inversion of mucosal entry may occur during closure, particularly when performed by beginners. To solve such clinical problems, we investigated a novel method for closure of a mucosal entry using the clip-with-line method.

First, a clip with line is left on the proximal side and another is left on the distal side of the mucosal entry. The line is pulled and mild tension is generated at the mucosal entry, which is subsequently lifted up. The clips are left from the distal to proximal sides of the mucosal entry. In this situation, precise clipping is very easy. The line is then cut after the closure of the mucosal entry (Fig. $1 \mathrm{~A}-\mathrm{F}$ ).

Using a monofilament line is favorable because it is easy to cut the line. The advantages of closure of mucosal entries using the clip-with-line method include avoidance of inversions of mucosal entries and slippage of clips, and low interindividual difference; furthermore, the technique is particularly useful for beginners. To date, several endoscopic suturing techniques during POEM have been reported [3-6]. The clip-withline method is useful for the closure of mucosal entries and does not require specialized instrumentation compared with conventional techniques.

\section{Acknowledgment}

The authors thank the patients and clinical staff for their participation and contribution to this study.

\section{References}

1. Inoue $\mathrm{H}, \mathrm{Minami} \mathrm{H}$, Kobayashi $\mathrm{Y}$, et al. Peroral endoscopic myotomy (POEM) for esophageal achalasia. Endoscopy 2010;42:265-271.

2. Shiwaku $\mathrm{H}$, Inoue $\mathrm{H}$, Yamashita $\mathrm{K}$, et al. Peroral endoscopic myotomy for esophageal achalasia: outcomes of the first over 100 patients with short-term follow-up. Surg Endosc 2016;30:4817-4826.

3. Shiwaku $H$, Inoue $H$, Yamashita Y. Mucostomy closure using the endoloop/clips technique in a purse-string manner after an
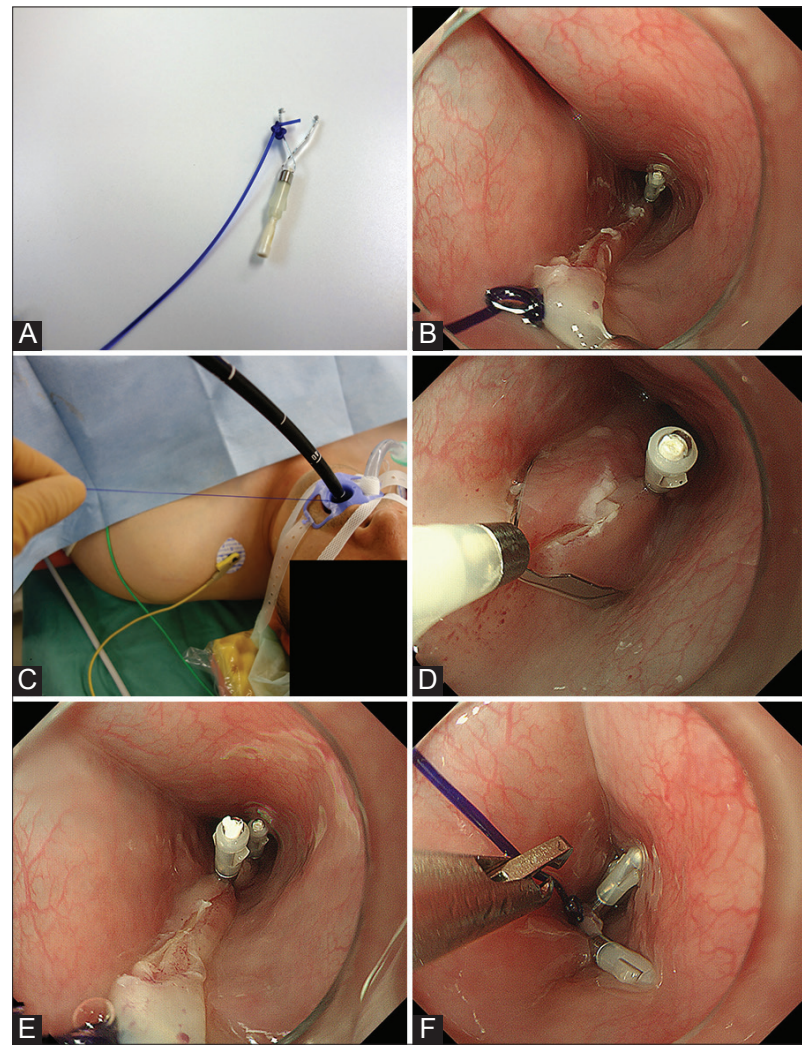

Figure 1 (A) A clip with line. (B, C) A clip-with-line is left on the proximal side and another on the distal side of the mucosal entry. The line is pulled, generating a mild tension at the mucosal entry, which is subsequently lifted up. (D, E) The clips are left from the distal to proximal sides of the mucosal entry. In this situation, precise clipping is very easy. (F) After closure of the mucosal entry, the line is cut

unsuccessful closure during peroral endoscopic myotomy. Dig Endosc 2015;27:630-631.

4. Saxena P, Chavez YH, Kord Valeshabad A, Kalloo AN, Khashab MA. An alternative method for mucosal flap closure during peroral endoscopic myotomy using an over-the-scope clipping device. Endoscopy 2013;45:579-581.

5. Li H, Linghu E, Wang X. Fibrin sealant for closure of mucosal penetration at the cardia during peroral endoscopic myotomy (POEM). Endoscopy 2012;44(Suppl 2 UCTN): E215-E216.

6. Yang D, Draganov PV. Closing the gap in POEM. Endoscopy 2013;45:677.

aDepartment of Gastroenterological Surgery, Faculty of Medicine, Fukuoka University, Fukuoka (Hironari Shiwaku, Kanefumi Yamashita, Suguru Hasegawa); ${ }^{b}$ Digestive Diseases Center, Showa University Koto Toyosu Hospital, Tokyo (Haruhiro Inoue), Japan

Conflict of Interest: None

Correspondence to: Hironari Shiwaku, MD, PhD, Department of Gastroenterological Surgery, Faculty of Medicine, Fukuoka University, Fukuoka, Japan, e-mail: hiro.shiwaku@gmail.com

Received 3 December 2017; accepted 11 December 2017; published online 26 January 2018

DOI: https://doi.org/10.20524/aog.2018.0230 